\title{
Multiple Embedding Using Robust Watermarks for Wireless Medical Images
}

\author{
Dominic Osborne and Derek Abbott* \\ Centre for Biomedical Engineering (CBME) \\ The University of Adelaide, SA 5005, Australia
}

\author{
Matthew Sorell and Derek Rogers \\ School of Electrical and Electronic Engineering \\ The University of Adelaide, SA 5005, Australia
}

\begin{abstract}
Within the expanding paradigm of medical imaging and wireless communications there is increasing demand for transmitting diagnostic medical imagery over error-prone wireless communication channels such as those encountered in cellular phone technology. Medical images must be compressed with minimal file size to minimize transmission time and robustly coded to withstand these wireless environments. It has been reinforced through extensive research that the most crucial regions of medical images must not be degraded and compressed by a lossless or near lossless algorithm. This type of area is called the Region of Interest (ROI). Conversely, the Region of Backgrounds (ROB) may be compressed with some loss of information to achieve a higher compression level. This type of hybrid coding scheme is most useful for wireless communication where the 'bit-budget' is devoted to the ROI. This paper also develops a way for this system to operate externally to the Joint Picture Experts Group (JPEG) still image compression standard without the use of hybrid coding. A multiple watermarking technique is developed to verify the integrity of the ROI after transmission and in the situation where there may be incidental degradation that is hard to perceive or unexpected levels of compression that may degrade ROI content beyond an acceptable level. The most useful contribution in this work is assurance of ROI image content integrity after image files are subject to incidental degradation in these environments. This is made possible with extraction of DCT signature coefficients from the ROI and embedding multiply in the ROB. Strong focus is placed on the robustness to JPEG compression and the mobile channel as well as minimizing the image file size while maintaining its integrity with the use of semi-fragile, robust watermarking.
\end{abstract}

Keywords: Semi-fragile Watermarking, Authentication, Medical Images.

\section{Introduction}

Increasingly medical images are acquired, stored and transmitted digitally. This is especially the case for digital images that are used in radiology [Osborne et al. 2002]. As these types of images are typically of large size, compression allows for the cost of storage to be reduced and the speed of transmission to be increased. Although the cost of transmission bandwidth is decreasing there remains a need of authentication for these types of images and provision for compression so that feasible transmission is possible. The use of a simple system that provides some compatibility with current image compression standards is essential as complex compression schemes are expensive to develop and deploy [Clunie 2000] and allow for very limited usage. This paper presents a technique that can be used to verify the integrity of medical images prior to any diagnosis that is made after transmission over a wireless link through the use of semi-fragile watermarking, which is robust to JPEG [Wallace 1991] compression. This has provided a level of assurance that important detail is present and has not been lost as a result of incidental degradation over a noisy channel. The type

*e-mail: dosborne@eleceng.adelaide.edu.au

Permission to make digital or hard copies of part or all of this work for personal or classroom use is granted without fee provided that copies are not made or distributed for profit or commercial advantage and that copies bear this notice and the full cit ation on the first page. To copy otherwise, to republish, to post on servers, or to redistribute to lists, requires prior specific permission and/or a fee.

MUM 2004, October 27-29, 2004 College Park, Maryland, USA.

Copyright 2004 ACM 1-58113-981-0/04/10 ...\$5.00 of image that will be used to highlight this type of detail includes a spiral hairline fracture, which is a classic example of this type of diagnostic detailed information. A special subset of authentication watermarking is implemented around the ROI into the ROB to provide authentication of these types of images. This can be extended to any image with a critically important region. As this type of watermarking specifically survives JPEG compression, transmission of a small image file is possible without sacrificing image quality in the ROI where no watermark is placed. This scheme can be applied in one of two ways illustrated in Fig. 1. The first operates externally to the JPEG standard and allows for the entire image to be compressed to specified level. This results in good bit rate performance consistent with typical levels of JPEG compression at the extent of some degradation of the ROI. The other scheme operates by embedding a watermark is an identical way, but encoding the DCT coefficients directly into a hybrid-coded JPEG-like file. This technique results in minimal degradation and near-lossless encoding of the ROI at the expense of a larger file size and slightly inferior bit-rate performance. This must function within the framework of the JPEG standard rather than operating as an external system.

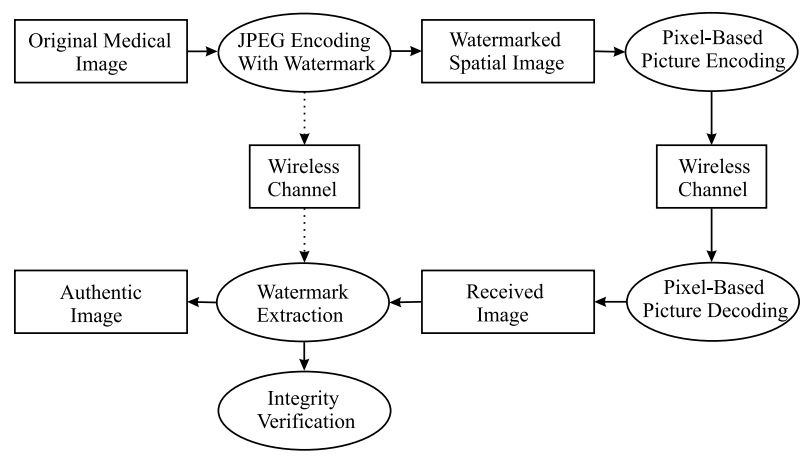

Figure 1: Two possible wireless image scenarios. The first transmission involves dual encoding of the ROI and ROB resulting in a larger file size and improved ROI quality, while the second transmission involves the complete lossy JPEG encoding of the entire image or any near-lossless pixel encoding methodology, such as GIF or JPEG-2000. 


\section{Problem Statement}

\subsection{The Problem of Authentication}

Adding small amounts of noise to corrupt the bitstream of an image file that has been channel-coded does not usually affect the importance of the diagnostic features present in the image after transmission has taken place. Incidental distortions that are not corrected through channel decoding [Viterbi 1967] may slightly distort the file structure of the compressed image file without any noticeable change to perceptual quality. This could involve a loss of diagnostic feature information, which for medical images is detrimental as detailed density information is mandatory. Hence it is critical to authenticate image quality prior to any diagnosis that is made [Osborne et al. 2003]. A classic example of this type of feature information is shown in the Infant's Fracture of Fig. 2.

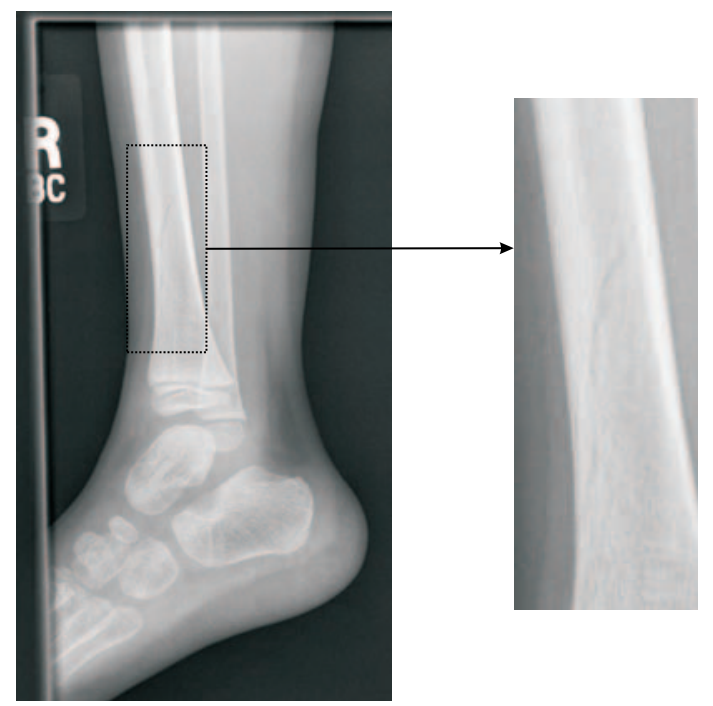

Figure 2: Non-displaced hairline fracture from the leg of an infant, which is often invisible on initial radiographs. If this type of image was transmitted from a hospital to a mobile hand-held device there would be an immediate need to evaluate image quality as a result of the possible loss of image transform coefficients in the ROI or unexpected levels of compression that might degrade feature content in this region.

\subsection{The Problem of Large File Size}

To maintain as much detail as possible, digital medical images are typically stored without loss of information using lossless compression schemes which allows for complete image restoration. The long term digital storage or mobile transmission of such images is prohibitive without the use of lossy image compression to reduce the image file sizes. As a typical example a mammogram may be digitized at $2048 \times 2048$ pixels at 16 Bits Per Pixel (bpp), leading to a file which is over 15 megabytes in size [Strom and Cosman 1997]. The use of lossless formats is widely accepted because no image information is discarded and data is interchangeable from one format to another. This simply leads to a different representation of the image file, but guarantees consistent visual appearance and diagnostic quality of the image. For widespread usage, lossy compression involves the use of JPEG standards. The most common of these which is implemented in most hardware is the well established Baseline JPEG. This involves performing the Discrete Cosine Transform (DCT) [Ahmed et al. 1974] on $8 \times 8$ image pixels to create micro-blocks, quantization of these coefficients and entropy coding of the result. Contrary to excellent developments in lossy image compression, these types of schemes are viewed with suspicion by many members of the scientific and medical community who believe that image alteration may lead to loss of diagnostic or scientific value.

\section{Previous Work in ROI Watermarking}

The concept of ROI watermarking was first proposed by [Wakatani 2002] who placed signature information into the ROB. A progressively compressed version of a signature image is used and the most significant information is embedded into the region closest to the ROI. This method allows for the signature image to be detected with moderate quality from a clipped version of the image that included the ROI. This system was intended for use over web-based medical image database systems with primary focus placed on ensuring copyright and intellectual property protection. The ROI area in the original image is specified prior to compressing the signature image using a progressive encoding algorithm to generate a bitstream. This allows for increasing visual detail with as the extracted bitstream is followed. The payload is embedded into pixels around the ROI in a spiral way as depicted in Fig. 3. Another re-

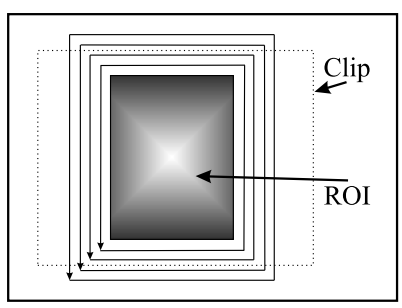

Figure 3: Since the ROI is the most critical aspect of a medical image, it may be clipped to include the ROI. The signature image is compressed using Embedded Zerotree Wavelet (EZW) coding so that the whole image can be reproduced with average quality and the entire signature image can be retrieved. The quality of the resulting signature image is directly correlated to the length of the bitstream extracted.

cent ROI watermarking scheme is proposed by [Lie et al. 2003], which is designed to operate within the framework of the JPEG2000 standard, targeting ROI compressed images. A dual watermarking scheme is proposed in which critical image content is to be authenticated. Two different types of watermarks are used, one being naturally fragile and the other robust. The embedding process for the robust watermark takes place at differing resolution layers to ensure that malicious changes are detected and provides flexibility in determining the extent of alteration to discriminate intended attacks from unintended ones. In order to accurately detect which areas have been altered, the first watermark $W 1$, which is sensitive and fragile is hidden in the ROI. The second watermark, $W 2$ is composed of features of mid-frequency wavelet sub-bands and is robustly watermarked into the ROB using features from the ROI as the signature shown in Fig. 4. The robust watermark proposed is designed to survive after acceptable levels of low-pass filtering and JPEG-2000 compression and not to survive malicious attacks. This signature is based on wavelet coefficient properties of the ROI, where features are extracted based on absolute differences between corresponding coefficients in the $L H 3$ and $H H 3$ subbands on $8 \times 8$ 


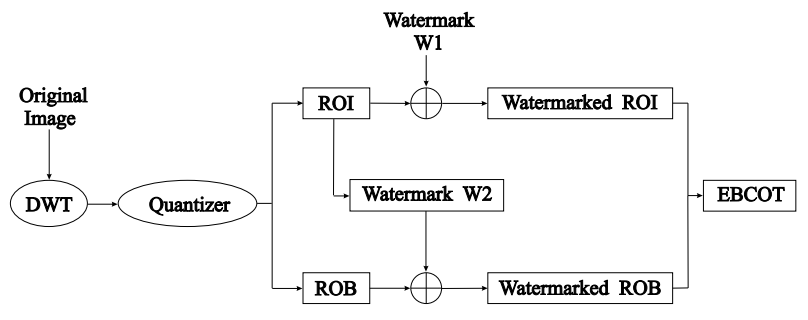

Figure 4: Block diagram of proposed dual watermarking scheme using ROI features as part a watermark to be robustly embedded in the ROB. This is designed to withstand typical levels of JPEG2000 compression. To be consistent with this standard, scalable image coding is used.

blocks. Similarly in this work a signature is based on on the absolute differences between corresponding coefficients in adjacent DCT tiles from inside the ROI, which have been uncorrelated as part of the signature extraction process. [Lie et al. 2003] mentions that his procedure degrades the ROB significantly, however this is not a primary concern as the ROB area is typically encoded at a low quality and gains minimal attention from users. The main focus of the works by [Lie et al. 2003] and [Wakatani 2002] is copyright protection and assurance that malicious attacks on the embedded watermarks are prevented. Our focus is primarily concerned with integrity verification as images are to be transmitted in error-prone lossy transmission channels, such as those encountered in mobile phone telephony to those in Wireless Local Area Networks (LANs.) The most useful contribution in our work is assurance of ROI image content integrity after image files are subject to incidental degradation in these environments. This is made possible with extraction of DCT signature coefficients from the ROI and embedding multiply in the ROB.

\section{Authentication Watermarking Technique Used}

If the signature information is lumped and localized within the ROB it is possible to authenticate and verify the diagnostic integrity of such images. A simple method to multiply watermark involves embedding in the same shape of the ROI in the eight regions surrounding the ROI or fewer regions if the space in the ROB is unavailable. A visual impression of this method is shown in Fig. 5. Multiple embedding can give the receiver additional confidence in the unlikely event that both a watermark and signature are corrupted in an identical way and the watermark is falsely detected as authentic. It may also be of benefit if one watermark is corrupted. Semi-fragile (or robust) watermarking is specifically designed to withstand application specific transformation operations, such as lossy compression and geometric distortions [Lin and Chang 2001], but is designed to be corrupted as a result of undesirable alterations such as malicious manipulations and incidental degradation over a mobile link which may or may not be perceptible to the receiver. Semi-fragile robust signature embedding ensures that the watermark survives JPEG compression or slight degradation up to a point where the value of the work is lost. Because ROI compression has been successfully subjectively evaluated in ROB of diagnostic medical images [Anastassopoulos and Skodras 2002], the radiologist can have greater confidence that the diagnostic value of the image has not been degraded.

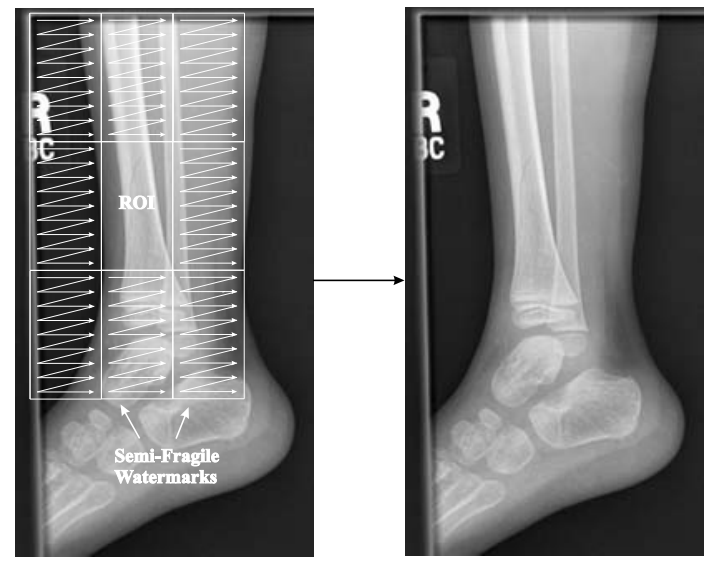

Figure 5: Multiple embedding in the ROB: The algorithm embeds a signature in the eight regions surrounding the ROI or in fewer regions if space is unavailable. Watermarking takes place following the direction of the arrows.

\subsection{Basis of Signature Extraction}

The basis of singular semi-fragile watermark extraction and embedding was initially developed by [Lin and Chang 2001]. Standard lossy image compression systems involve converting an image into some transform domain, such as wavelet or block DCT domain and quantizing the coefficients in order to reduce their entropy. Coefficients are quantized to a level proportional to how easy it is to perceive changes in them and the property of quantization of coefficients is exploited to remove redundancy in the image. Let $x \bullet q$ be the result of quantizing $x$ to an integral multiple of a quantization step size, $q$ :

$$
x \bullet q=q\left[\frac{x}{q}+0.5\right] .
$$

Consider $s$ to be a real valued scalar quantity and $q_{1}$ and $q_{2}$ as quantization step sizes with $q_{2} \leq q_{1}$, then

$$
\left(\left(s \bullet q_{1}\right) \bullet q_{2}\right) \bullet q_{1}=s \bullet q_{1} .
$$

If $s$ is quantized to an even multiple of the larger step $q_{1}$ and then by a smaller step $q_{2}$, the effect of the second quantization can be reversed. The watermark should survive as long as the quantization that is performed during compression uses smaller step sizes. The watermark embedding and extraction procedure is designed to survive typical levels of JPEG compression, where images are quantized in the block DCT domain. The quantization step size for each coefficient depends on its frequency. These step sizes are obtained by multiplying the transform coefficients by a predefined quantization table, which is scaled by a constant. A signature is extracted from the low frequency terms of the micro-blocks of the ROI and embedded into the high frequency terms of the ROB as a semifragile watermark, which is illustrated on a block level in Fig. 6. This is important as the low-frequency terms represent the most important picture information that cannot be degraded through incidental degradation. High frequency coefficients can be used for the embedding in the peripheral regions, as these areas are diagnostically less important and will be degraded through compression. A signature for the image is extracted by converting the medical image into its $8 \times 8$ block DCT representation and grouping blocks of the image into pseudo-random pairs according to a specified seed. For each pair of DCT blocks, 8 corresponding low frequency coefficients are compared to obtain 8 bits of the binary signature. Con- 


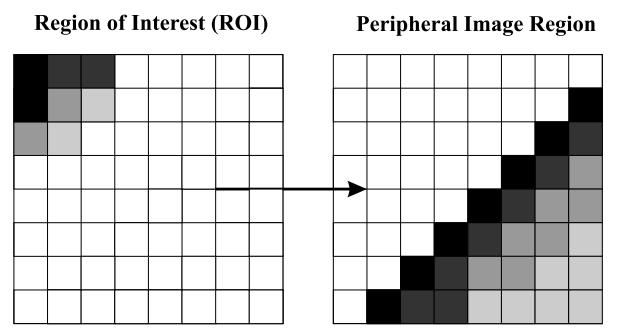

Figure 6: Spatial representation of the bits used for the signature (left) from the ROI and the bits used for the watermark (right) corresponding to the peripheral regions. Matching shades indicate where the payload bits go.

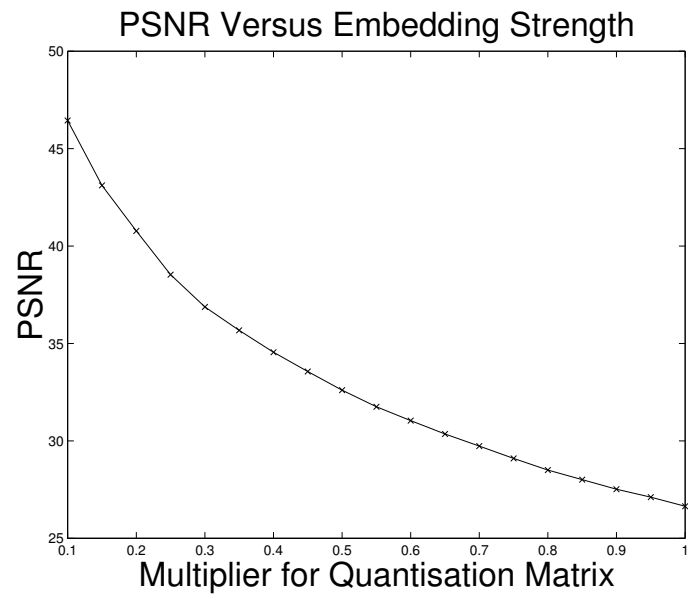

Figure 7: Deterioration of image quality with increasing embedding strength with resulting distortion quantified with the Peak Signal to Noise Ratio (PSNR). This is to be expected and is unavoidable as robust watermarking is used, which is nearly always perceptible to the observer.

sider two blocks that have been grouped $C_{a}$ and $C_{b}$, then:

$$
\text { signature bit }=\left\{\begin{array}{lll}
0 & : & C_{a}[i, j]<C_{b}[i, j] \\
1 & : & C_{a}[i, j] \geq C_{b}[i, j]
\end{array}\right.
$$

where $i$ and $j$ are the coordinates of a low frequency coefficient from Fig. 6. So that the reader can have some perspective on the extent of image degradation resulting from varying embedding strengths of watermarking. A hundred randomly selected grayscale images were tested for their Peak Signal to Noise Ratio (PSNR) after using embedding took place, as seen in Fig. 7. The greater the embedding strength, the more compression the image can survive and the more perceptible the watermark will be. This is not a problem as removal of the watermark can be performed easily at the receiving end.

\section{System Implementation of Dual Encod- ing Scheme}

The main sub-systems used in the systematic design of the ROI semi-fragile watermarking scheme designed to work within the framework of JPEG are illustrated in Fig. 8. The image undergoes a block-based DCT specified by a tile or block size, which is typically $8 \times 8$ pixels. These coefficients are rounded and quantized and

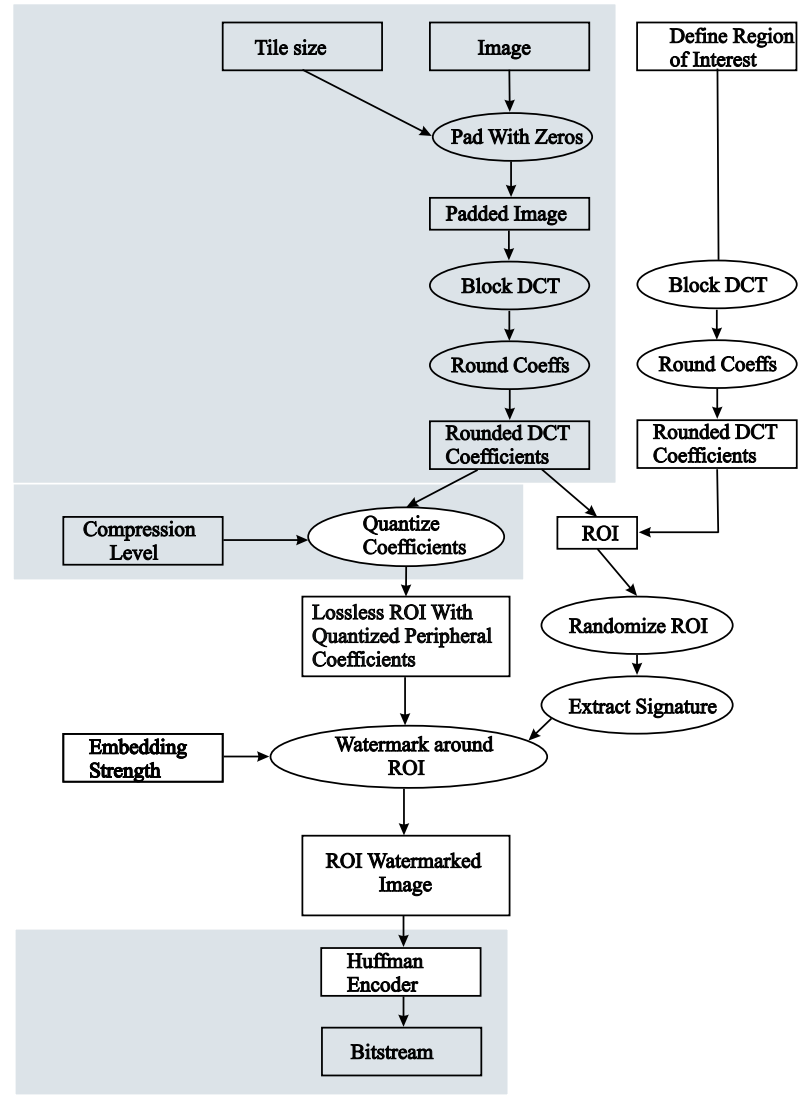

Figure 8: Dual encoding scheme designed to work within the framework of the JPEG standard. A ROI is specified and copied from an image that then undergoes a block-based DCT and quantization to minimize the number of non-zero coefficients for the purposes of high compression resulting in improved bit-rate performance. The compression level is specified by the user as a quantization table multiplier. Sub-systems standard to JPEG are shaded in grey.

entropy [Huffman 1962] encoded. Those areas not shaded in grey include operations within the framework of the standard that can be used for more accurate ROI integrity verification than the system that operates externally to JPEG. The ROI in its transform representation replaces the same region in the full image whose coefficients have been quantized. This ensures that the ROI is stored nearlosslessly while the ROB is compressed using lossy JPEG compression and contains at least one watermark.

\section{Performance through robustness testing}

Survival of JPEG compression is one of the primary requirements of the ROI watermarking scheme. This is mandatory if operation external to JPEG is required, where the pixel-based image compression method is treated as part of the communication channel as shown in the flow diagram of Fig. 1, ignoring the wireless channel on the left side of this diagram. The watermarked image is permitted to undergo types of lossless compression, which will not degrade the image pixels or lossy JPEG, which can be applied up to a threshold specified by the user by the embedding strength. Robustness to varying levels of JPEG compression took place on 100 grayscale images of arbitrary types and varying resolutions from 


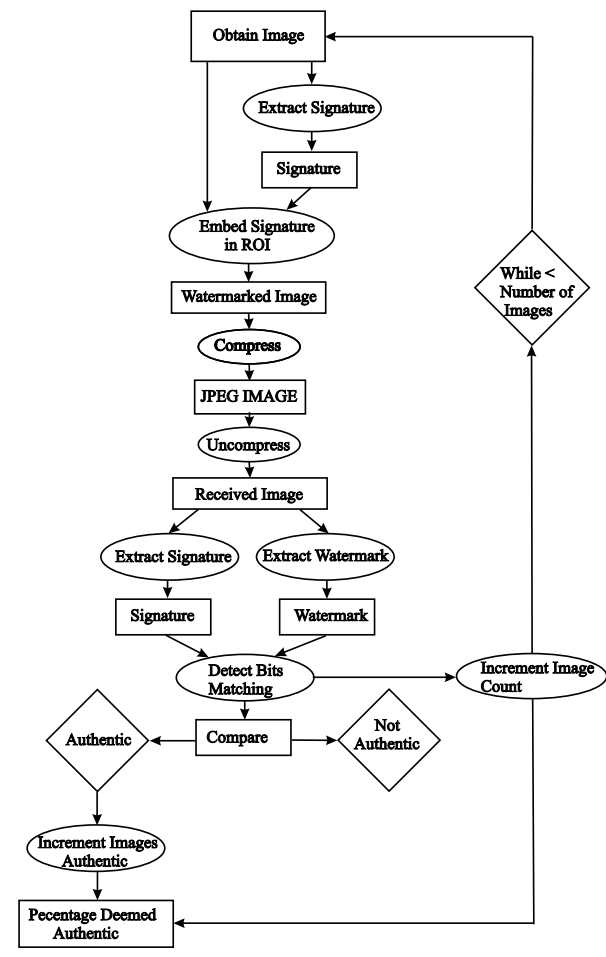

Figure 9: System to test the robustness of ROI watermarking system. The signature extracted from the ROI is embedded within the transform domain of the image. This is subjected to varying degrees of JPEG compression and converted back to a spatial representation. The signature and the watermarks are extracted and compared to authenticate the image. To accommodate minimal bit errors resulting from conversion from transform to spatial representation a 5 percent error is permissable on comparison of extracted signature with extracted watermark for a match.

$256 \times 256$ to $1280 \times 1280$ pixels. The ROI was specified to occupy a sufficiently small area at the center of each image so that 8 watermarks could be embedded around this region. Results are shown in Fig. 9. The results of this test shown in Fig. 10 demonstrate that the ROI watermarking scheme survives JPEG compression levels up to and exceeding the watermark embedding strength used on $90 \%$ of the images. This is shown to be consistent for three typical JPEG compression levels. These results are almost identical to those obtained by [Cox et al. 2002] where the performance of a similar watermarking method was tested where a signature was extracted from an image and a singular watermark embedded in the same region. As the scheme developed involves embedding a signature into the same coefficients in $8 \times 8$ DCT transform blocks, it is expected to survive similar levels of compression resulting in correlating sets of results. Approximately $10 \%$ of the images do not survive JPEG compression for quantisation levels exceeding the embedding strength. This problem can easily be rectified by setting the embedding strength slightly above the level of required JPEG compression.

\section{Bit-rate Performance of Hybrid Coding Scheme}

If the image is sufficiently robustly watermarked and converted to spatial domain for complete JPEG compression, the resulting file

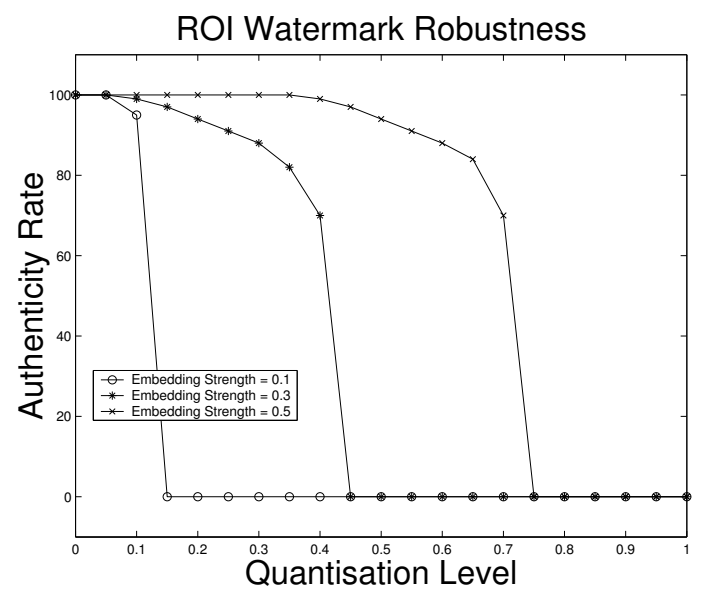

Figure 10: Testing the robustness of 100 images with the semifragile ROI watermarking scheme designed to withstand JPEG compression. As expected, the system fails the authentication test consistently after each of the three watermark embedding levels.

size is directly related to the level of compression. This results in a bit-rate performance that is identical to JPEG. If degradation of the ROI through JPEG quantization is not permissable and hybrid coding is preferred as illustrated in the flow diagram of Fig. 8 the bulk of the 'bit budget' will be stored in the ROI. This is because quantisation does not take place in this region and all ROI transform coefficients must be encoded, which are typically non-zero. As the ROB can undergo compression through quantisation, the majority of coefficients will be zero. This will result in a file size that is dependent on the size of the selected ROI. The larger this region is, the more near-lossless compression is required, the larger the file size. For a typical fracture or tumor, the area of the ROI does not typically extend beyond $20 \%$ of the entire image. This is also verified in work by [Foos et al. 2000] and [Anastassopoulos and Skodras 2002] where ROI Maxshift JPEG-2000 compression was utilized to compress these types of medical images. Strom [Strom and Cosman 1997] also validated the effectiveness of combined lossy and lossless JPEG compression with these types of ROI sizes. It was shown through extensive subjective testing that the diagnostic value of the medical image did not degrade for very low bit rate coding. These approaches reinforce that the ROI is exactly the area where all diagnostic information is located. Bit-rate performance was evaluated in Fig. 11 with and without the use of watermarking and with sizes of ROI varying from complete lossy compression, where the peripheral regions were the entire image to the extreme of having the entire image encoded near-losslessly as a ROI. The most practically applicable areas of these curves includes those areas up to and around having a 20 percent area devoted to the ROI. Within this area of the curve the use of one or more embedding regions does not significantly affect the size of the medical image file. It would appear that each embedding region in Fig. 5 increases the file size by approximately $0.1 \mathrm{bpp}$, which without watermarking results in a compression level of $2 \mathrm{bpp}$. The increase in file size is insignificant in comparison with complete near-lossless JPEG encoding that provides minimal compression of $5 \mathrm{bpp}$ of the original grayscale image. After baseline JPEG compression, which corresponds to using a quantization multiplier of 1 , it is typical for the resulting quantized $8 \times 8$ DCT coefficient ROB blocks to be reduced from 64 to an average of 2 coefficients. This confirms the majority of the bit-budget is devoted to the ROI, which makes the compression scheme useful for low bandwidth mobile communication. This does assume that the ROI chosen is relatively small in comparison 


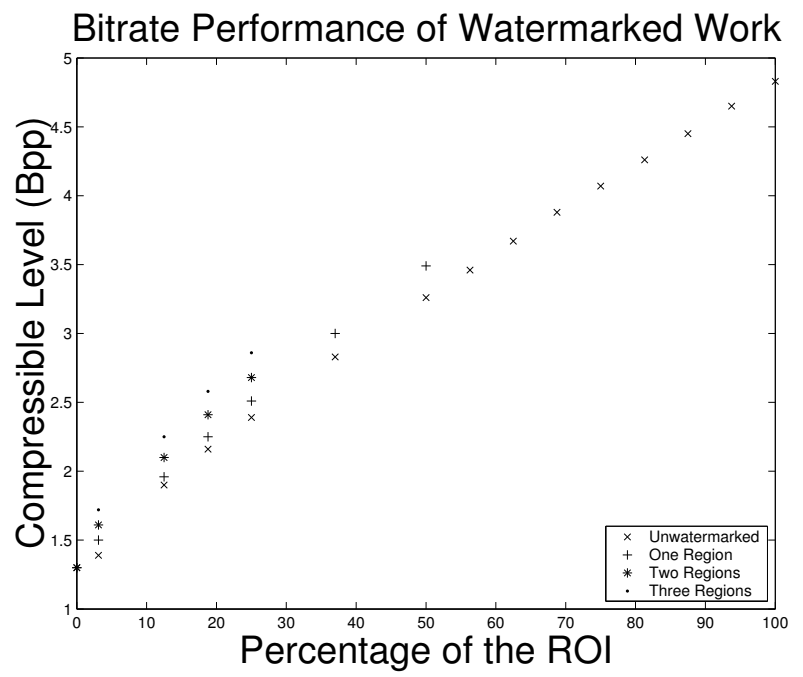

Figure 11: Bit-rate performance which can be compared with entire lossy JPEG compression, where the area of the ROI is zero to entire lossless compression in which the percentage of the ROI is 100 .

to the rest of the image.

\section{Conclusion}

The use of watermarking can be used to verify the integrity of digital images. A method is developed which is designed to be used on images that have critically important regions. The scheme is designed to perform multiple watermarking around the ROI containing features from this region. This technique can provide assurance that this region has not been corrupted as a result of incidental degradation resulting from a wireless link or that the ROI has not exceeded a compression level threshold. As watermarked images can be compressed, a smaller file size is achievable. This facilitates verification of ROI integrity as well as wireless communication. The method used allows the user to evaluate quality of this region in a received image without the need of a reference image. This is most useful for transmitted medical images where high levels of quality assurance are mandatory prior to making any diagnosis. The technique used can be systematically designed in two ways, one which fits in a modular way into the JPEG standard resulting in minimal changes and hybridly coding the ROI and the ROB resulting in superior ROI quality. Alternatively this method can operate extraneously to the standard providing greater compliance and improved bit-rate performance. This results in a degraded ROI when lossy JPEG is used on the watermarked image pixels or improved picture quality if lossless picture encoding techniques are used. This method could also be used to monitor image or video quality for quality control systems or benchmarking image/video processing systems and algorithms. A limitation is that authentication based on watermarking cannot replace classical cryptographic authentication protocols that protect communication channels. Embedded robust watermarking for ROI integrity verification can allow for compression and provision of image integrity. This can be most useful for medical images that must be transmitted quickly in a wireless environment.

\section{References}

Agrafiotis, D., Bull, D. R., And Canagarajah, N. 2003. Region of interest coding of volumetric medical images. In IEEE International Conference on Image Processing (ICIP), vol. 2, 217-220.

Ahmed, N., Natarajan, T., And Rao, K. R. 1974. Discrete cosine transform. IEEE Transactions on Computer Theory C-23 (January), 90-94.

AnAstassopoulos, G. K., AND SkOdRAs, A. 2002. JPEG2000 ROI coding in medical imaging applications. In Proceedings of the 2nd IASTED International Conference on visualisation, imaging and image processing (VIIP2002).

Clunie, D., 2000. Lossless compression of greyscale medical images - effectivness of traditional and state of the art approaches.

Cox, I. J., Miller, M. L., ANd Bloom, J. A. 2002. Digital Watermarking, 1 ed. The Morgan Kaufmann Series in Multimedia Information and Systems. Morgan Kauffman Publishers, 340 Pine St, Sixth Floor, San Fransisco, CA 94104-3205, USA.

Foos, D. H., Muka, E., Slone, R. M., Erikson, B. J., Flynn, M. J., Clunie, D. A., Hildebrand, L., Kohm, K., AND YounG, S. 2000. JPEG2000 compression of medical imagery. 85-96.

HUfFMAN, D. A. 1962. A method for the construction of minimum redundancy codes. Proceedings of the IEEE 40, 10981101.

LIE, W.-N., HSU, T.-L., AND LIN, G.-S. 2003. Verification of image content integrity by using dual watermarking on wavelets domain. In IEEE International Conference on Image Processing (ICIP), vol. 2, 487-490.

Lin, C. Y., AND Chang, S. F. 2001. A robust image authentication method distinguishing JPEG compression from malicious manipulations. IEEE Transactions on Circuits and Systems of Video Technology 11, 2, 153-168.

Osborne, D., Авbott, D., Coutts, R., And Rogers, D. 2002. An overview of wavelets for image processing for wireless communications. In SPIE Smart Structures, Devices and Systems, vol. 4935, 427-435.

Osborne, D., Abbott, D., Sorrell, M., And Rogers, D. 2003. Embedded importance watermarking for image verification in radiology. In SPIE BioMEMS and Nanotechnology, vol. 5275, 383-390.

Penedo, M., Peraman, W. A., Tahoces, P. G., Souto, M., AND VIDAL, J. J. 2003. Embedded wavelet region-based coding methods applied to digital mammography. In IEEE International Conference on Image Processing (ICIP), vol. 2, 197-200.

Strom, J., AND Cosman, P. 1997. Medical image compression with lossless regions of interest. Signal Processing 3, 155-171.

VITERBI, A. J. 1967. Error bounds for convolutional codes and an asymptotically optimum decoding algorithm. IEEE Transactions on Information Theory IT-13 (April), 260-269.

WAKATANI, A. 2002. Digital watermarking for ROI medical images by using compressed signature image. In Proceedings of the 35th Hawaii International Conference on System Sciences. IEEE Computer Society, vol. 6, 157-163.

WALlaCE, G. K. 1991. The JPEG still picture compression standard. Communications of the ACM 34, 4 (April), 30-45. 